\title{
Valoraciones subjetivas y condiciones objetivas de confort térmico en viviendas
}

\section{Subjective evaluations and objective conditions of thermal comfort in residential architecture}

Gabriela Arrieta

Universidad Nacional de Córdoba (UNC), Facultad de Arquitectura Urbanismo y Diseño (FAUD), Centro de investigaciones Acústicas y luminotécnicas (CIAL), Consejo Nacional de Investigaciones Cientificas y Técnicas (CONICET), Córdoba, Argentina

e-mail: g.arrieta@live.com.ar

\section{RESUMEN}

El siguiente artículo, describe el estudio realizado en un conjunto de viviendas de la ciudad de Córdoba, con el objetivo de comprender como las personas perciben el ambiente térmico de sus viviendas. Partiendo de una investigación doctoral en curso, basada fundamentalmente en el confort higrotérmico de espacios interiores residenciales bajo la concepción adaptativa, y valorando la idea de que el paradigma de confort ha ido evolucionando en los últimos años, el interés de este artículo es describir las sensaciones subjetivas referidas al confort higrotérmico de las personas que habitan en Córdoba. El estudio metodológico se desarrolló a través del método comparativo constante, recurriendo a entrevistas guiadas y a la sensibilidad teórica del investigador. Las conclusiones abordadas tienen que ver con el impacto negativo que produce la incomodidad térmica en la vida de las personas y la dependencia inconsciente a la tecnología para alcanzar confort, en lugar de estrategias pasivas.

\section{ABSTRACT}

The following article describes the study carried out in a group of houses in the city of Córdoba, with the aim of understanding how people perceive the thermal environment of their homes. Starting from an ongoing doctoral research, based mainly on the hygrothermal comfort of residential interior spaces under the adaptive conception, and assessing the idea that the comfort paradigm has evolved in recent years, the interest of this article is to describe the sensations subjective related to the hygrothermal comfort of the people who live in Córdoba. The methodological study was developed through the constant comparative method, using guided interviews and the theoretical sensitivity of the researcher. The conclusions addressed have to do with the negative impact that thermal discomfort produces in people's lives, the unconscious dependence of the user on technology to achieve comfort, instead of passive strategies.

PALABRAS CLAVE: Acondicionamiento - Climatización - Residencial.

KEYWORDS: Conditioning - Air Conditioning - Residential.

FECHA DE RECEPCIÓN: 27/08/2020 | FECHA DE ACEPTACIÓN: 22/10/2020

DOI: http://dx.doi.org/10.30972/arq.0164553 


\section{INTRODUCCIÓN}

Lo que la sociedad piensa y valora en relación al confort higrotérmico, ha ido cambiando con el paso del tiempo y a través de la historia. La palabra CONFORT por definición, hace referencia a contener y reforzar, aquello que produce bienestar; pero en el siglo XVII este concepto era referido a lo privado o doméstico, luego en el XVIII hacía referencia a la comodidad y el ocio; en el XIX a la calidad de los elementos mecánicos que proveen luz, calor o ventilación; entrando en el siglo XX esta palabra hizo referencia a la eficiencia y la comodidad. Hasta que se empezó a estudiar considerando los factores internos y externos que influyen en la sensación de confort. Es necesario comprender la importancia del bienestar térmico, como menciona Nicol, "por tres razones: poder proveer condiciones satisfactorias a las personas, controlar el consumo de energía y sugerir y establecer estándares". (Nicol, 1993, citado por , Taleghani, et.al., 2013, p.202).

Cada persona es un sujeto que está constituida en sujeción a otros, el colectivo. En el concepto de confort, se involucran aspectos que tienen que ver con este sujeto único como son los factores personales; pero también otros factores que consideran al colectivo, como son los factores socioculturales. Esta investigación, indaga sobre la sensación subjetiva de confort valorada por los usuarios, en la cual intervienen aspectos biológicos, psicológicos y fisiológico en relación al entorno climático particular que en este caso es la ciudad de Córdoba.

A pesar de la gran cantidad de definiciones que existen sobre este concepto, se define en el marco de este trabajo al Confort higrotérmico como la ausencia de malestar térmico, cuando los mecanismos fisiológicos termorreguladores del cuerpo no necesitan intervenir. Esta sensación de comodidad surge de la generación de un microclima que evita la reacción del cuerpo ahorrando gastos de energía que en otro caso utilizaría para lograr el estado térmico deseado, mecanismo involuntario conocido como termorregulación natural. Esta capacidad innata de adaptación al medio desarrolla (Canul, 2018) en su tesis, describiendo las formas más importantes relacionadas al intercambio térmico entre el cuerpo humano y el medio ambiente: convección, que refiere a los intercambios de calor con el aire; radiación, al intercambio con las superficies del entorno y los intercambios de calor latente debido a la evaporación del sudor. Gracias a estos mecanismos, el ser humano puede logar acercar el ambiente térmico real al deseado para alcanzar el confort. Un ambiente es calificado como confortable, cuando no hay presencia de incomodidad o malestar térmico alguno. Es decir, que el usuario de dicho ambiente no sienta demasiado calor o frio. Respecto a este concepto (Canul, 2018,p.36), agrega en su investigación que "Si la temperatura de la piel cae por debajo de los $34^{\circ} \mathrm{C}$, los sensores de frío envían impulsos al cerebro que activará los mecanismos de regulación. Si, por el contrario, la temperatura interior excede los $37^{\circ} \mathrm{C}$, será el sensor de calor en el hipotálamo quien enviará los impulsos. La evaluación del ambiente térmico se basará en las señales de estos dos sistemas de sensores".

Los factores o parámetros de confort son aquellas condiciones propias del lugar que inciden en las sensaciones de los ocupantes, y por lo tanto, en sus valoraciones respecto de este ambiente. Hay factores ambientales como son la temperatura, humedad, velocidad del aire, etc; factores personales que se refieren a metabolismo, nivel de arropamiento, edad, genero, estado de salud, historial térmico, tiempo de permanencia, etc., y factores socioculturales que tienen que ver con expectativas de confort, motivaciones, procedencia, acceso a la tecnología, economía, políticas de estado, etc. Dicho confort térmico es un objetivo importante del entorno construido, ya que determina gran parte del grado de satisfacción de sus ocupantes, su salud y su productividad. (Kim et al., 2018). Como se menciona anteriormente, no sólo depende de factores ambientales; también de las condiciones biológicas, fisiológicas y psicológicas del ser humano que 
experimenta el ambiente. Es por ello que su cuantificación resulta compleja, subjetiva y variable (Nematchoua et. al., 2014) citado por (Jara, 2015).

Según aseguran (Gómez-Azpeitia, Bojórquez Morales, y Pavel Ruiz Torres, 2007), la determinación de una temperatura ideal de diseño resulta en todos los casos el eje fundamental en la toma de decisiones. ¿Cómo puede determinarse esa temperatura en que las personas se sientan en confort, a fin de diseñar, construir y operar los edificios de la manera más sustentable posible? Jara, (2015) describe que en la actualidad, hay dos enfoques enfrentados que responderían a esta pregunta. Por un lado, el enfoque cuantitativo que toma variables ambientales y personales (unificadas o estandarizadas en cámaras de laboratorio) y desestima la variable subjetiva. Surge de este enfoque el Voto Medio Previsto, que establece o predice como se sentirán las personas en un ambiente térmico determinado. Estos estudios en cámaras climáticas, permitieron conclusiones como las descriptas por Canul, (2018,p.13), donde refiere el estudio realizado por Yu et al (2012), donde concluyen que "las personas en ambientes con ventilación natural tienen una aclimatación mayor a cambios fuertes de temperatura en comparación con las personas usuarias de ambientes con aire acondicionado, de tal manera que estas últimas ven disminuida su capacidad de adaptación a los ambientes cálidos actuales y futuros". Por otro lado, se encuentra el enfoque cualitativo, el cual considera la adaptación de las personas, las variaciones de la temperatura exterior y subjetividades, surgiendo así el modelo adaptativo. Canul, (2018,p.14) en su tesis describe que "el fin práctico de los estudios de campo de confort térmico, ha sido el desarrollo de estándares de confort a nivel local que sean aplicables durante el diseño y construcción de las edificaciones. En ellos se consideran factores sociales, culturales, condiciones de climatización, pero principalmente factores del clima local."

Las cuatro revoluciones industriales, llevaron a que mentalmente y de forma inconsciente, todos nos adaptemos a depender de la tecnología. Mientras más urbana y más rica en recursos económicos es la sociedad, más altas son las expectativas de confort que pretende alcanzar y conservar. Con este concepto, se observa que desde los años 60 hasta la actualidad, las preferencias de confort han ido evolucionando para las personas. Hablando de un paradigma de confort en constante cambio (Arrieta y Maristany, 2018), "La sociedad actual exige lugares seguros, limpios y bien climatizados, para lo que es necesario integrar percepciones y exigencias de los habitantes y alcanzar un óptimo equilibrio entre estándares sociales, uso de la energía y desarrollo sostenible."(Vargas y Pulgarín Gallego, 2005, p.1).

\section{METODOLOGIA}

En esta investigación, se busca comprender, como perciben el ambiente térmico los habitantes de un grupo de viviendas con condiciones espaciales y formales equivalentes. Se recurre a la llamada teoría fundamentada, la cual exige del investigador identificar categorías teóricas que son derivadas de los datos mediante la utilización de un método comparativo constante creado por Glaser \& Strauss (1967). Dicho Método comparativo constante (M.C.C.), es una metodología de investigación cualitativa para la recolección y análisis de datos empíricos destinados a producir conocimiento científico. Recurre a la sensibilidad teórica del investigador, y exige comparar contenidos de diversas entrevistas o de observaciones y relevamientos con los conceptos teóricos nacientes del esfuerzo de identificar los temas fundamentales y recurrentes expresados por los sujetos encuestados.

La teoría fundamentada, es utilizada para investigaciones cualitativas ayudando a comprender con gran claridad los fenómenos de carga subjetiva. A través de esta forma de investigación, se puede detectar cuáles son los aspectos relevantes en un área de estudio específica, haciendo una revisión exhaustiva de experiencias verbalmente expresadas por los informantes. Dicho método (M.C.C), fue elegido para esta investigación por tratarse de variables en primer lugar 
desconocidas para el investigador, por lo que se necesita la subjetividad de los encuestados, y en segundo lugar por ser una investigación de aspectos cualitativos que se requieren descubrir con el aporte de los usuarios y la observación del investigador. La iniciativa de este estudio, surge de la curiosidad por conocer como sienten las personas que habitan en la Ciudad de Córdoba el ambiente térmico de sus viviendas.

Las viviendas estudiadas, se encuentran localizadas en Córdoba, Argentina, Barrio La Salle, dentro de la zona nor-oeste de la mancha urbana. Se trata de un grupo de viviendas construidas a fines de los años ' 80 por el Banco Hipotecario, bajo el concepto de viviendas sociales para clase media. Siendo iguales en tipología, disposición sobre el eje sur/norte (con el frente al sur y patio al norte), ubicadas entre medianeras, un amplio retiro verde en el frente y patio trasero. Techos de losa maciza inclinados a $45^{\circ}$ en estar y dormitorios y techo plano en el sector de baño y cocina. Paredes de bloque de cemento de $20 \mathrm{~cm}$ revocado por fuera y bolseado por dentro.

Las mediciones se realizaron en los espacios de estar mientras se llevaban adelante las entrevistas guiadas a los habitantes de las viviendas, intentando agotar las respuestas de cada pregunta para lograr obtener diferentes datos. En primer lugar, las respuestas de los habitantes se registran completas a través de grabaciones, simultáneamente las palabras claves son registradas de forma escrita por el encuestador y las percepciones del propio investigador en el momento de la entrevista. Al mismo tiempo, se relevan los datos cuantitativos de temperatura y humedad con un termómetro de globo marca Lutron modelo WBGT-2010SD. El encuestador, realiza las preguntas orientadas al confort higrotérmico interior a los habitantes, y se dio especial atención a cada respuesta que abra una posibilidad de ampliar el tema. Dicha entrevista constó de 14 preguntas que el encuestador fue haciendo a cada sujeto encuestado, a medida que éste iba relatando sus respuestas:

1. ¿Cómo describirías tu sensación al encontrarte en la vivienda?

2. ¿Cuánto tiempo pasa en tu vivienda al día?

3. ¿Qué es lo que piensas respecto de la temperatura interior de tu vivienda?

4. ¿Qué es lo que piensas respecto de la humedad interior de tu vivienda?

5. ¿Qué opinas respecto de tu confort higrotérmico dentro de tu vivienda?

6. ¿Cómo opinas que esta situación fue cambiando desde que habitas esta vivienda?

7. ¿Cómo cambiarias la situación que ofrece tu vivienda para alcanzar comodidad térmica?

8. ¿Cómo relacionas las condiciones interiores de tu vivienda con las exteriores?

9. ¿Cómo cambia esta relación en los distintos momentos del año?

10. ¿Hay algún momento del año donde sientas que las condiciones son favorables?

11. ¿Utiliza las posibilidades de su vivienda para mejorar las condiciones higrotérmico interiores (ventanas, postigos, cortinas, etc.)?

12. ¿Cómo se vincula tu comodidad térmica con el consumo eléctrico de tu vivienda?

13. ¿Cómo siente que influye la temperatura interior de su vivienda en su arropamiento durante los distintos momentos del año?

14. ¿Cómo cree que debería modificarse su vivienda para responder a sus necesidades de confort?

Se entrevistaron a las familias residentes en las 5 viviendas seleccionadas. 7 Hombres y 6 mujeres de entre 30 y 60 años de edad, en buen estado general de salud que residen las viviendas de forma permanente hace varios años. El estudio se llevó adelante en un mismo día durante el invierno y un día durante el verano. Hasta que las respuestas empezaron a ser recurrentes y repetitivas, lo que definió de forma clara que era momento de terminar el estudio de campo y comenzar a procesar los datos obtenidos. Se analizaron los dos periodos por separado y luego se hicieron algunas reflexiones generales. 


\section{DESARROLLO}

El clima de la ciudad de Córdoba; se define mayormente como templado y cálido, con inviernos benignos y veranos poco calurosos. En términos generales es templado moderado con las cuatro estaciones bien definidas. Los inviernos no son muy fríos y poco lluviosos. Los veranos son húmedos, con días calurosos y noches frescas. La normativa de referencia que se utiliza en el diseño y la construcción dentro de la ciudad, es la norma IRAM, específicamente la IRAM 11603, (1996), define las diferentes zonas climáticas o bioambientales del país y para cada una de ellas establece características climáticas comunes y algunos consejos sobre pautas de diseño a tener en cuenta. En esta norma, se considera a la ciudad en estudio, dentro de la zona bioambiental III a (Clima Templado Cálido), con amplitudes térmicas de más de $14^{\circ} \mathrm{C}$. Mientras que la zona III b es templada cálida con amplitudes térmicas menores de $14^{\circ} \mathrm{C}$. En la Tabla 1 se muestran los datos climáticos generales indicados por la norma.

Tabla 1: Datos clima Ciudad de Córdoba

\begin{tabular}{|c|c|c|c|cc|c|cc|c|ccc|c|c|c|}
\hline \multicolumn{10}{|c|}{ INIERO } \\
\hline LAT & LONG & ASIM & TMED & TMAX & TMIN & TMA & TDMN & PREC & HR & HELRE & VM & GD18 & GD 18 & GD 20 & GD22 \\
\hline-31.4 & 84.18 & 425 & 13.48 & 19.9 & 7.1 & -5.2 & 2.9 & 45 & 88 & 5.8 & 4.2 & 483 & 738 & 10.88 & 1528 \\
\hline \multicolumn{10}{|c|}{} \\
\hline LAT & LONG & ASIM & TMED & TMAX & TMIN & TMA & TDMN PREC & HR & HELRE & VM & GD18 & GD 18 & GD20 & GD22 \\
\hline-31.4 & 84.18 & 425 & 23.65 & 29.8 & 17.8 & 41.0 & 38.2 & 493 & 88 & 7.8 & 5.0 & & & & \\
\hline
\end{tabular}

Fuente: IRAM 11603.(IRAM 11603, 1996).

Se reconocen los requisitos para que la arquitectura sea adaptada a los distintos climas, son claras las recomendaciones y normas para lograr de forma pasiva el confort térmico de las personas en un ambiente determinado; con múltiples referenciales, como en las IRAM, donde se especifica para cada zona bioambiental y sus características, el criterio constructivo a considerar para cada orientación. $Y$ en el caso de que, siguiendo estos criterios normativos, igualmente no se alcance el estado de confort deseado, también se conocen las acciones internas o externas, voluntarias o involuntarias para acercar el ambiente térmico real al deseado.

Ciertamente, el problema radica en que prima una tendencia de exigir parámetros más extremos de los que se necesitan para estar biológicamente confortables (Arrieta y Maristany, 2018). Hay una tendencia generalizada por regular los niveles de climatización mecánicos de los espacios interiores a temperaturas muy frías en verano y muy calientes en invierno. Haciéndose imposible de alcanzar a través de los mecanismos pasivos de acondicionamiento conocidos que evitarían el colapso energético. Es así, que el resultado es el uso intensivo y cada vez mayor de sistemas de acondicionamiento artificial. Por consiguiente, se incrementa el consumo energético de las ciudades; que son cada vez más densas, lo cual aumenta aún más el consumo. La crisis energética es una de las mayores preocupaciones actuales que compete cada vez a más países, inclusive a los que se encuentran en vías de desarrollo (Balter et al., 2013). Razón por la cual, el adecuado acondicionamiento de los espacios interiores juega un rol fundamental en el desarrollo sostenible.

Dentro del conjunto de viviendas tomado como caso de estudio, en la calle Daniel Conci (Figura 1), todas las viviendas fueron construidas exactamente iguales en localización respecto del centro, orientación, dimensiones, alturas, materiales, técnica constructiva y uso. Con el paso del tiempo, varias viviendas fueron modificando algunas condiciones; ampliando habitaciones, anexando otras o agregando /quitando aberturas, parasoles o postigos. 


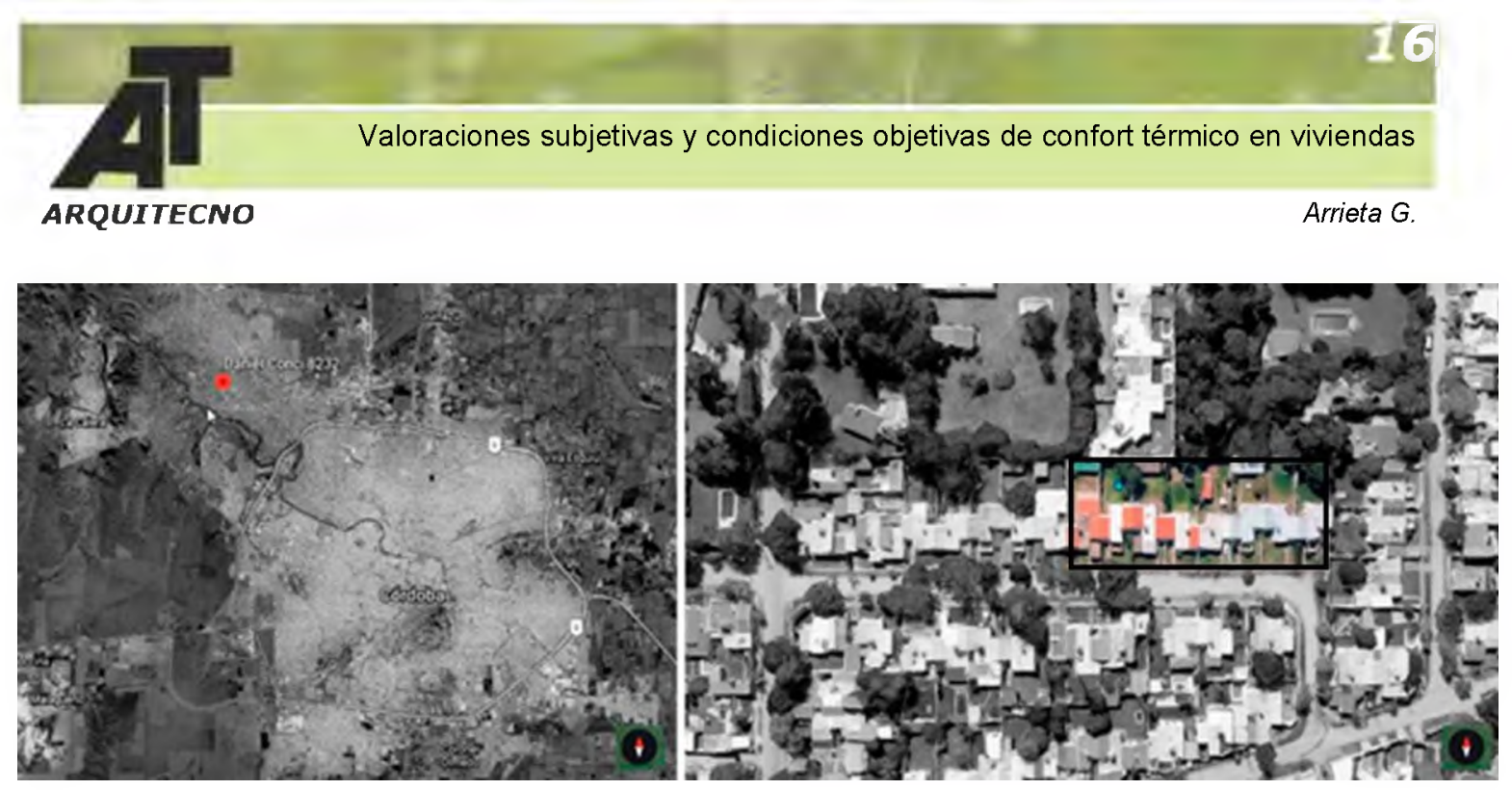

Figura 1: Ubicación del sitio de estudio en relación a la mancha urbana de Córdoba Capital. Imagen de la calle, marcando las viviendas consideradas para el estudio. Imagen extraída de Google Earth, 2020.

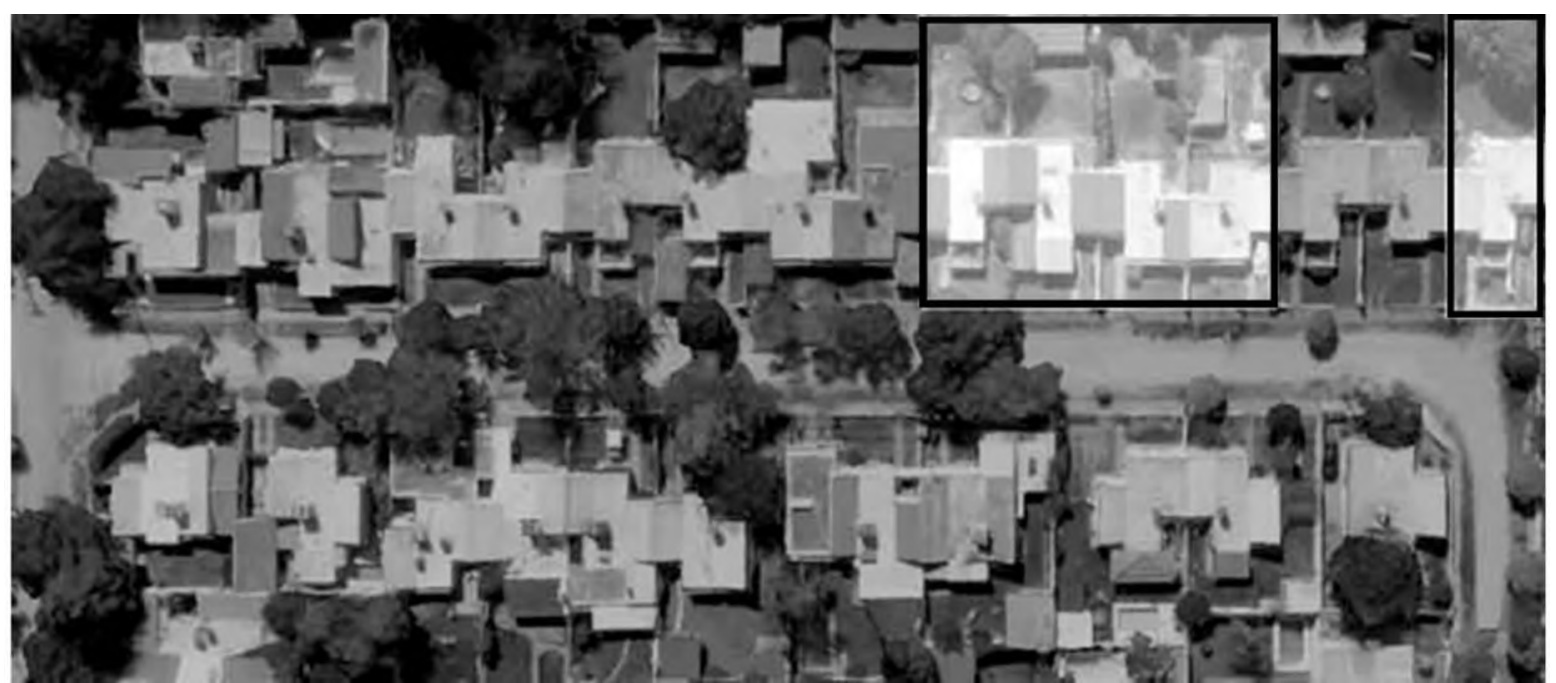

Figura 2: Calle Daniel Conci y destacadas las viviendas donde se realizó el estudio con el M.C.C. Imagen extraída de Google Earth, 2020.

En la Figura 2 se marcan las viviendas en las cuales se realizó el estudio, (aquellas iluminadas), se seleccionaron teniendo en cuenta que se mantienen originales, sin alteraciones en sus dimensiones, cantidad de ambientes, materiales, alturas, usos, etc.

\section{RESULTADOS DEL M.C.C. REALIZADO}

El día estudiado del periodo de invierno fue seleccionado por las particularidades climáticas del mismo. Se eligió un día de buen asoleamiento y poco viento, como también, el momento de inicio luego de las 10 de la mañana. Realizadas varias entrevistas a los vecinos de las viviendas seleccionadas, se detectaron algunos problemas percibidos recurrentemente, que se mencionan a continuación:

- $\quad$ Alta humedad interior

- Vivienda fría en invierno

- Dependencia de calefacción

- Poco uso de adaptación pasiva (envolventes)

Teniendo en cuenta los valores medidos por el termómetro de globo, en simultáneo con las entrevistas, se observa que las temperaturas de todas las viviendas se mueven entre los $16.5^{\circ} \mathrm{C}$ a los $19.5^{\circ} \mathrm{C}$ (Tabla 3). Es decir que son ciertamente frías o sobre el límite de lo valorado como confortable de acuerdo a los índices establecidos por las diferentes teorías indicadas en la tabla 2 . 
Tabla 2: Zona de confort establecida por fuentes de referencia

\begin{tabular}{|l|l|l|l|l|l|l|l|l|}
\hline \multicolumn{9}{|l|}{ Verano } \\
\hline Olgyay & $21,5^{\circ} \mathrm{C}$ & $27,5^{\circ} \mathrm{C}$ & $30 \%$ & $60 \%$ & $20{ }^{\circ} \mathrm{C}$ & $24{ }^{\circ} \mathrm{C}$ & $30 \%$ & $60 \%$ \\
\hline Givoni & $18^{\circ} \mathrm{C}$ & $23{ }^{\circ} \mathrm{C}$ & $20 \%$ & $80 \%$ & $21^{\circ} \mathrm{C}$ & $26{ }^{\circ} \mathrm{C}$ & $20 \%$ & $80 \%$ \\
\hline Evans & $18^{\circ} \mathrm{C}$ & $28{ }^{\circ} \mathrm{C}$ & \multicolumn{2}{|l|}{ Amplitud de 0 a $8{ }^{\circ} \mathrm{C}$} & $18^{\circ} \mathrm{C}$ & $28{ }^{\circ} \mathrm{C}$ & Amplitud de 0 a $8{ }^{\circ} \mathrm{C}$ \\
\hline IRAM & $22{ }^{\circ} \mathrm{C}$ & $27{ }^{\circ} \mathrm{C}$ & $50 \%$ & $55 \%$ & $18^{\circ} \mathrm{C}$ & $21^{\circ} \mathrm{C}$ & $50 \%$ \\
\hline
\end{tabular}

Fuente: Elaboración propia.

En invierno, el problema más recurrente en los usuarios entrevistados es la alta humedad interior, y es significativo que, en todos los relevamientos, las condiciones indicadas por el termómetro están dentro de las definidas como confortables, alrededor del $65 \%$ (Tabla 3). Por otro lado, el estudio se hizo en el periodo de invierno, pero como se mencionó anteriormente, en un día particularmente soleado. Se debe destacar, que, en todas las viviendas, las ventanas y postigos estaban cerrados, y en algunas incluso estaba encendida la calefacción. Habiendo podido mejorar las condiciones de confort con la radiación solar que ofrecía el exterior, las personas eligieron recurrir al acondicionamiento mecánico en primera instancia.

Durante el periodo de verano, se escogió un día en el que las temperaturas se esperaban no muy altas y la nubosidad era abundante. Con la misma guía de preguntas, en enero se volvió a entrevistar a los mismos usuarios. $Y$ se detectaron algunos problemas percibidos recurrentemente en el periodo estival, como se mencionan a continuación:

- Alta humedad interior

- Viviendas calurosas

- Poco uso de cortinas, postigos y ventanas

- Necesitad de AACC.

En verano, el problema más recurrente fue también la alta humedad interior, y nuevamente los valores del termómetro de globo marcaban valores dentro de lo establecido por la norma como confortable, rodeando el $58 \%$. Las temperaturas relevadas en las viviendas fueron alrededor de los $28^{\circ} \mathrm{C}$ o $29^{\circ} \mathrm{C}$ (Tabla 3). Es decir, unos pocos grados por encima de lo valorado como confortable por las diferentes teorías (Tabla 2). Por otro lado, la mayoría de las viviendas no tenían abiertas las ventanas para favorecer la ventilación cruzada o el recambio de aire que permitían las condiciones exteriores. Habiendo podido mejorar las condiciones de forma pasiva, se menciona la necesidad de encender o instalar un equipo de aire acondicionado, aunque todos los encuestados notaban y lamentaban el impacto que estas formas de climatización tenían en sus consumos de energía y los consecuentes costos económicos. 
Tabla 3: Resumen por vivienda de los resultados obtenidos en el estudio M.C.C. de invierno y verano. Respuestas de los encuestados, observaciones del investigador y registros emocionales.

\begin{tabular}{|c|c|c|c|c|c|}
\hline \multicolumn{3}{|l|}{ INVIERNO } & \multicolumn{3}{|c|}{ VERANO } \\
\hline ENTREVISTADOS & $\begin{array}{c}\text { OBSERVACIO } \\
N\end{array}$ & REGISTRO & ENTREVISTADOS & OBSERVACION & REGISTRO \\
\hline \multicolumn{6}{|c|}{ VVIENDA 1} \\
\hline $\begin{array}{l}\text { cuando me levanto siento frio, luego me aclimato. En } \\
\text { inviemo necesito calefacción. Hay mucha humedad. } \\
\text { Pero se tolera. Nos vamos acostumbrando. En verano } \\
\text { con ventilador esta bien. A la mañana hace mas fno, a } \\
\text { la tardecita se calienta. No uso los parasoles o } \\
\text { ventanas por costumbre. }\end{array}$ & $\begin{array}{l}\text { Casa luminosa, } \\
\text { ventanas y } \\
\text { postigos } \\
\text { cerrados, sin } \\
\text { calefacción. }\end{array}$ & \begin{tabular}{|l|} 
comodidad \\
térmica, luz \\
natural, día \\
soleado. $70,6 \%-$ \\
$16.5^{\circ} \mathrm{c}$ \\
\end{tabular} & $\begin{array}{l}\text { Es térmicamente estable. Sólo algunos dias } \\
\text { se siente mucho el calor. Nos vamos } \\
\text { adaptando, mucha ventilación cruzada. } \\
\text { AACC solo para dormir. En inviemo es } \\
\text { cálido, en verano suele ser fresca. En verano } \\
\text { usamos poca ropa. }\end{array}$ & $\begin{array}{l}\text { casa muyiluminada. Sin } \\
\text { cortinas. Ventilador } \\
\text { encendido. Short y ojotas. } \\
\text { Abiertos postigos y } \\
\text { ventanas. }\end{array}$ & $\begin{array}{l}\text { Agradable. } \\
\text { Afuera } \\
\text { nublado. } \\
58.4 \%-28.9^{\circ} \mathrm{C}\end{array}$ \\
\hline \multicolumn{6}{|c|}{ VIVIENDA 2 } \\
\hline $\begin{array}{l}\text { estamos cómodos. Es tirando a fresca, pero nos } \\
\text { gusta. No consumimos acondicionamiento, ni nos } \\
\text { abrigamos mucho. Queremos poner cortinas. }\end{array}$ & $\begin{array}{l}\text { Casa luminosa, } \\
\text { postigos } \\
\text { cerrados, sin } \\
\text { corriente de } \\
\text { aire. } \\
\end{array}$ & $\begin{array}{l}\text { cómodo, luz, día } \\
\text { soleado. } 64.4 \% \text {. } \\
18,7^{\circ} \mathrm{C}\end{array}$ & $\begin{array}{l}\text { Casi siempre se siente bien, un poco de } \\
\text { humedad en los placares al sur. Las cortinas } \\
\text { van a ayudar. No usamos mucho AACC, de } \\
\text { noche a veces. }\end{array}$ & $\begin{array}{l}\text { lluminado, sin cortinas } \\
\text { ventilador prendido. } \\
\text { Musculosa, vestido y } \\
\text { ojotas }\end{array}$ & $\begin{array}{l}\text { agradable, luz, } \\
\text { bienestar, } \\
58,8 \%-28,9^{\circ} \mathrm{C}\end{array}$ \\
\hline \multicolumn{6}{|c|}{ VIVENDA 3} \\
\hline $\begin{array}{l}\text { Siempre estoy cómoda, de noche es un frízer. } \\
\text { Muchísi ma humedad, es terrible. Mejoro con el gas } \\
\text { natural, siempre esta calentito ahora. Si o si } \\
\text { consumimos mucho en calefacción. La humedad te } \\
\text { mata. }\end{array}$ & $\begin{array}{l}\text { Todo cerrado, } \\
\text { MUY cálido, } \\
\text { calefacción al } \\
\text { máximo. }\end{array}$ & $\begin{array}{l}\text { Calor, algo de } \\
\text { luz. Día soleado } \\
64,5 \%-18,5^{\circ} \mathrm{C}\end{array}$ & $\begin{array}{l}\text { La casa se siente bien, muchísima humedad. } \\
\text { Usamos ventanas, postigos y ventilador } \\
\text { SIEMPRE prendido. Usamos ropa muy } \\
\text { liviana. }\end{array}$ & $\begin{array}{l}\text { Ventanas cerradas, luz } \\
\text { apagada, ventilador } \\
\text { prendido. Rcpa liviana. }\end{array}$ & $\begin{array}{l}\text { Mas agobiante } \\
\text { que afuera. Dia } \\
\text { nublado. } \\
59,7 \%-29.4^{\circ} \mathrm{C}\end{array}$ \\
\hline \multicolumn{6}{|c|}{ VNIENDA 4} \\
\hline $\begin{array}{l}\text { El problema de estas casas es la humedad. Suele } \\
\text { estar calentito. Andamos muy abrigados igual. La } \\
\text { humedad es terrible. }\end{array}$ & $\begin{array}{l}\text { Cerrado, muy } \\
\text { cálido, } \\
\text { calefacción } \\
\text { encendida. }\end{array}$ & $\begin{array}{l}\text { Agobiante, algo } \\
\text { de luz natural, dia } \\
\text { soleado. } 67,1 \%- \\
18.0^{\circ} \mathrm{C}\end{array}$ & $\begin{array}{l}\text { La sensación térmica es horrible. Lo que mas } \\
\text { se siente es la humedad. La temperatura no } \\
\text { se siente tanto. Vamos a poner AACC } \\
\text { porque no se aguanta. Los problemas de } \\
\text { humedad se sienten todo el año. }\end{array}$ & $\begin{array}{l}\text { Ventanas cerradas, calor, } \\
\text { ventilador prendido. Ropa } \\
\text { liviana y escasa. }\end{array}$ & $\begin{array}{l}\text { Mas calor que } \\
\text { afuera. Dia } \\
\text { nublado. } \\
\text { interior oscuro. } \\
57 \%-29.4^{\circ} \mathrm{C}\end{array}$ \\
\hline \multicolumn{6}{|c|}{ VVIENDA 5} \\
\hline $\begin{array}{l}\text { Se siente mucho el frio. Usamos muchísimo el } \\
\text { calefactor, siempre!. No me importa el consumo, gasto } \\
\text { mucho en calefacciones. La humedad es terrible. } \\
\text { Influye la orientación sur. }\end{array}$ & $\begin{array}{l}\text { Todo cerrado, } \\
\text { bastante } \\
\text { caluroso, moho } \\
\text { en el techo. }\end{array}$ & $\begin{array}{l}\text { Oscuro, } \\
\text { apagada, sin sol, } \\
\text { afuera esta } \\
\text { cálido. } 55,3 \% \text { - } \\
19.0^{\circ} \mathrm{C}\end{array}$ & $\begin{array}{l}\text { Cuando hace frio o calor afuera, en la casa } \\
\text { también. Falta mucha aislación, usamos } \\
\text { mucho AACC, las ventanas poco. La } \\
\text { humedad es terrible. Es notorio el consumo } \\
\text { de gas, en estas casas te moris de frio. }\end{array}$ & $\begin{array}{l}\text { casa oscura, sin } \\
\text { acondicionamiento. } \\
\text { Musculosa y short. }\end{array}$ & $\begin{array}{l}\text { Pesado, } \\
\text { penumbras, } \\
\text { afuera caen } \\
\text { gotas y } \\
\text { refresca. } \\
57.7 \%-29.6^{\circ} \mathrm{C}\end{array}$ \\
\hline
\end{tabular}

Fuente: Elaboración propia.

\section{REFLEXIONES FINALES}

La incomodidad térmica impacta de forma negativa en las personas, y cuando los valores de temperatura están muy sobre los límites de lo teóricamente confortable, la humedad juega un rol fundamental en las valoraciones de los habitantes. El usuario promedio, exige con el paso del tiempo espacios interiores mejor climatizados y tiene menos tolerancia a la incomodidad térmica. Frente a una creciente demanda de confort térmico en ambientes interiores residenciales, y los problemas que esta tendencia trae consigo, como lo es el consumo excesivo de energía, generalmente eléctrica; dando su real importancia al creciente y constante calentamiento global, y considerando que el ser humano tiene de forma innata la capacidad biológica de adaptarse a climas variados y sus fluctuaciones temporales. Se abre un panorama que busca su respuesta desde la arquitectura y más específicamente, desde la correcta utilización de la envolvente.

En climas de grandes amplitudes térmicas, como es el caso de Córdoba donde la temperatura varía durante todo el año más de $14^{\circ} \mathrm{C}$ diarios, y donde las estrategias de diseño de las envolventes y de climatización pasiva no son tan rigurosas ni difundidas como en los climas más extremos, se debería aprovechar esta condición para el acondicionamiento pasivo de los espacios interiores. 

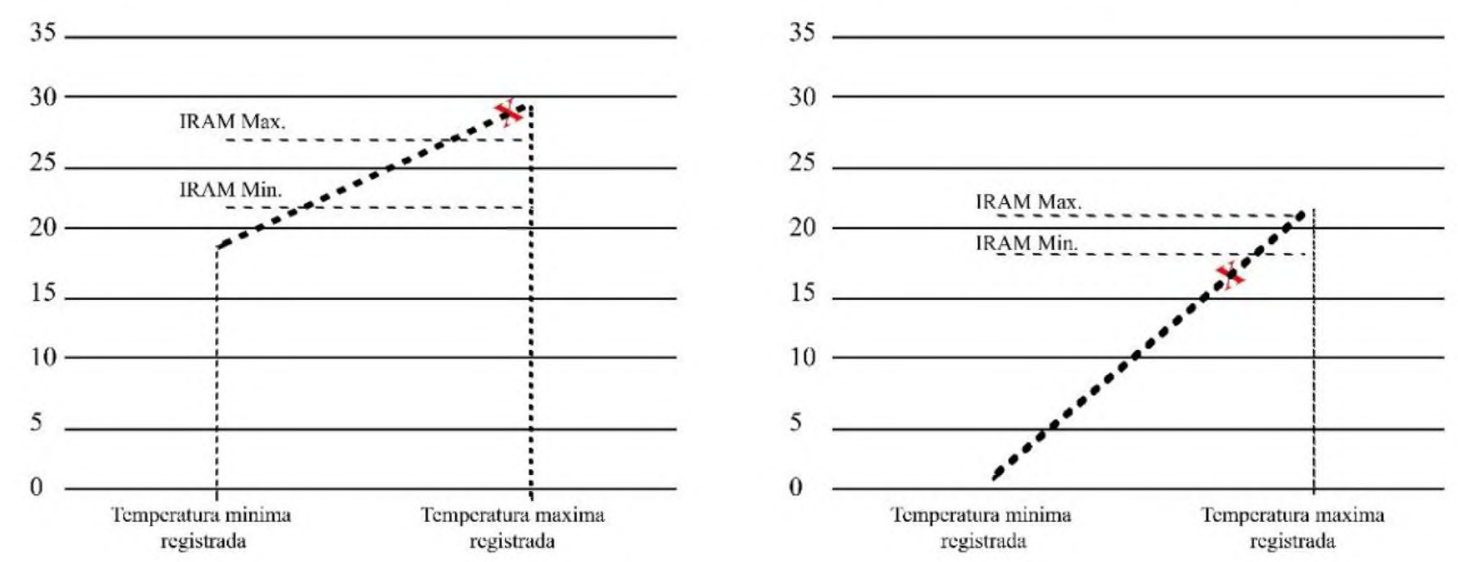

Figura 3: Amplitud térmica diaria exterior en ambos días de estudio, relación con las referencias mínimas y optimas lineales que propone IRAM y el momento de la medición concretamente (en rojo). Elaboración propia.

El estudio con el M.C.C se realizó tanto en el periodo de invierno como en el de verano; y en ambos, las variaciones de temperatura diarias fueron de $12^{\circ} \mathrm{C}$ y $22^{\circ} \mathrm{C}$ respectivamente (Figura 3), una condición que no se logra aprovechar si no es a través de la operabilidad criteriosa de las envolventes. Si se considera solo la condición aislada en el momento del estudio, ciertamente son condiciones de temperatura desfavorables, que avalan las sensaciones de los usuarios encuestados. Pero si se consideran las variaciones exteriores de ambos días de estudio, se puede ver que hubieran posibilitado mejorar las condiciones interiores significativamente y de forma pasiva. Ciertamente, el estudio realizado y presentado en este artículo fue breve, el periodo de tiempo estudiado y la muestra de usuarios seleccionada hacen que no sea posible generalizar los resultados. Pero se logran aclarar y confirmar algunas percepciones y comportamientos de los usuarios que sirven como disparador para futuras investigaciones.

En climas donde las condiciones exteriores alcanzan temperaturas frescas durante la noche y cálidas durante las horas más asoleadas, debe aprovecharse la envolvente de forma criteriosa y eficiente para lograr condiciones interiores aceptables por los usuarios. Con el método empleado en esta investigación, se logró detectar que el mayor problema mencionado por los usuarios fue la alta humedad interior. A pesar de no relevar temperaturas muy alejadas de los límites confortables establecidos por los autores referenciados, la humedad relativa interior jugo un rol preponderante acentuando los niveles de frio o calor percibidos por los usuarios. Siendo la humedad interior más percibida incluso que la temperatura que rozaba y sobrepasaba lo teóricamente confortable.

La percepción del confort interior es una vivencia subjetiva compleja, que solo puede medirse valorando un conjunto de aspectos simultáneos. Sobre todo, en los climas templados, los criterios constructivos para el confort térmico interior no son tan estandarizados como en climas más extremos, pero toma gran importancia la posibilidad de los usuarios de operar a través de las envolventes. Posibilitando mantener las condiciones interiores dentro de lo confortable por mayores periodos de tiempo y aprovechando las variaciones térmicas exteriores. 


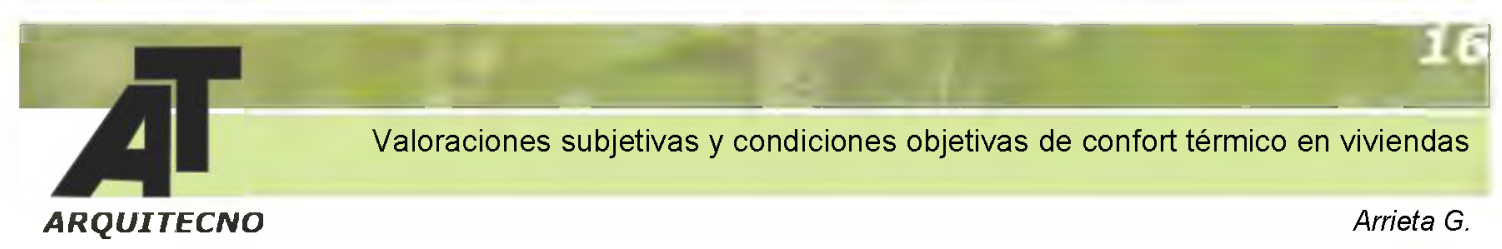

\section{REFERENCIAS BIBLIOGRÁFICAS}

Arrieta, G., \& Maristany, A. (2018). Cambiando los paradigmas: Revision del concepto de confort higrotermico desde los 60' hasta la actualidad. Averma, 22(2314-1433), 01.01-01.12.

Balter, J., Ganem, C., \& Discoli, C. (2013). Auditoría térmico-energética y percepción de confort en edificios en altura másicos y livianos en la ciudad de mendoza. Avances En Energías Renovables y Medio Ambiente, 1, 79-88.

Glaser, B., \& Strauss, A. The discovery of grounded therory strategies for qualitative research. New York: Aldine Publishing Company 1967.

Gómez-Azpeitia, G., Bojórquez Morales, G., \& Pavel Ruiz Torres, R. (2007). El confort térmico: dos enfoques teóricos enfrentados/Thermal comfort: two confronted theoretical focuses. Palapa, 2(1870-7483),

$45-57$.

http://revistasacademicas.ucol.mx/index.php/palapa/article/view/43

IRAM 11603. (1996). Acondicionamiento térmico en edificios. Clasificación bioambiental de la República Argentina. Norma Argentina.

Jara, P. (2015). Thermmal comfort and its importance for the architectural design and enviromental quelity of indoors spaces. UTOPIATEORIARAXIS, 7, 106-121.

Kim, J., Schiavon, S., \& Brager, G. (2018). Personal comfort models - A new paradigm in thermal comfort for occupant-centric environmental control. Building and Environment, January. https://doi.org/10.1016/j.buildenv.2018.01.023

Luis Armando Canul Euan. (2018). Método para determinar el rango de confort térmico estándar en espacios interiores para climas de méxico. Universidad de Colima.

Taleghani, M., Tenpierik, M., Kurvers, S., \& Van Den Dobbelsteen, A. (2013). A review into thermal comfort in buildings. Renewable and Sustainable Energy Reviews, 26, 201-215. https://doi.org/10.1016/j.rser.2013.05.050

Vargas, M. F., \& Pulgarín Gallego, I. (2005). Calidad ambiental interior: Bienestar, confort y salud. Rev Esp Salud Pública, 2, 243-251. 